\title{
Studies of the photoionization cross sections of $\mathrm{CH}_{4}$
}

\author{
M. Braunstein and V. Mckoy \\ Arthur Amos Noyes Laboratory of Chemical Physics, ${ }^{\text {) }}$ California Institute of Technology, Pasadena, \\ California 91125 \\ L. E. Machado \\ Departamento de Fisica, Universidade Federal de São Carlos, São Carlos, SP, Brazil \\ L. M. Brescansin \\ Instituto de Fisica, Universidade Estadual de Campinas, Campinas, SP, Brazil
}

M. A. P. Lima

Instituto de Estudos Avancados, CTA/FTE, C. P. 6044, Săo José dos Campos, SP, Brazil

(Received 11 April 1988; accepted 24 May 1988)

\begin{abstract}
We present cross sections and asymmetry parameters for photoionization of the $1 t_{2}$ orbital of $\mathrm{CH}_{4}$ using static-exchange continuum orbitals of $\mathrm{CH}_{4}^{+}$to represent the photoelectron wave function. The calculations are done in the fixed-nuclei approximation at a single internuclear geometry. To approximate the near-threshold behavior of these cross sections, we assumed that the photoelectron spectrum is a composite of three electronic bands associated with the Jahn-Teller components of the distorted ion. The resulting cross sections reproduce the sharp rise seen at threshold in the experimental data and are in good agreement with experiment at higher energy. The agreement between the calculated and measured photoelectron asymmetry parameters is, however, less satisfactory.
\end{abstract}

\section{INTRODUCTION}

The high symmetry of the $\mathrm{CH}_{4}$ molecule with its $1 a_{1}^{2} 2 a_{1}^{2} 1 t_{2 x}^{2} 1 t_{2 y}^{2} 1 t_{2 z}^{2}$ electron configuration suggests that many of the electronic properties of its valence orbitals could be thought of qualitatively and, to some extent, quantitatively in terms of a neon-like model. ${ }^{1}$ In fact, models using scaled $2 p$ atomic orbitals were used earlier to estimate the photoionization cross section of the $1 t_{2}$ orbital of methane. ${ }^{2}$ Available experimental data ${ }^{3,4}$ shows, however, that the $2 p$ and $1 t_{2}$ cross sections of $\mathrm{Ne}$ and $\mathrm{CH}_{4}$, respectively, are significantly different both in shape and magnitude. For example, the $1 t_{2}$ cross section of methane rises steeply near threshold and reaches a peak value four to five times larger than that of neon and falls off rapidly with photoelectron energy. The $2 p$ photoionization cross section of neon, in contrast, behaves quite differently. These features are illustrated in Fig. 1(a).

We have recently extended the iterative Schwinger variational method, ${ }^{5}$ which has been used to study the photoionization cross sections and photoelectron angular distributions of many linear molecules, ${ }^{6}$ to certain classes of nonlinear molecules including systems such as $\mathrm{H}_{2} \mathrm{O}, \mathrm{CH}_{4}$, $\mathrm{C}_{2} \mathrm{H}_{4}$, and $\mathrm{C}_{2} \mathrm{~F}_{2} \mathrm{H}_{2}{ }^{7}$ In this paper we present the results of applications of this method to study the photoionization cross sections and photoelectron angular distributions of the $1 t_{2}$ orbital of $\mathrm{CH}_{4}$. These cross sections and asymmetry parameters are obtained in both the length and velocity approximations using static-exchange frozen-core continuum orbitals of $\mathrm{CH}_{4}^{+}$to represent the photoelectron wave function. The calculations are done in the fixed-nuclei approximation and at one internuclear geometry. These cross sections agree well with those obtained by Cacelli et al. ${ }^{8}$ using a one-center representation of the molecular wave function

\footnotetext{
Contribution No. 7768
}

and the Stieltjes imaging technique, again with just functions centered on carbon. Experimental data on the $1 t_{2}$ orbital, ${ }^{9}$ however, shows that the Jahn-Teller effect plays a role in near-threshold structure of the photoionization spectrum of $\mathrm{CH}_{4}$. To approximate this behavior near threshold, we used our calculated fixed-nuclei cross sections at a single tetrahedral geometry but assumed that the photoelectron spectrum is a composite of three electronic bands associated with the Jahn-Teller components of the ${ }^{2} T_{2}$ ion of $\mathrm{CH}_{4}$ with respective maxima at $13.5,14.5$, and $14.8 \mathrm{eV} .{ }^{9}$ The Franck-Condon factors associated with these Jahn-Teller distortions can be expected to lead to a sharp rise in the cross section near threshold. The calculated cross sections agree well with the measured values of Backx et al. ${ }^{3}$ For example, they reproduce the sharp increase and prominent, but broad, maximum seen in the experimental cross sections within $5 \mathrm{eV}$ of threshold. Experiments with adequate photoelectron kinetic energy resolution to resolve vibrational structure within these Jahn-Teller components near threshold could provide considerable insight into the photoionization dynamics of $\mathrm{CH}_{4}$. The agreement between the calculated and measured photoelectron angular distributions ${ }^{10}$ is, however, less satisfactory.

For purposes of comparison, we have also obtained cross sections and photoelectron angular distributions for the $2 p$ orbital of neon using the same numerical procedures. Our calculated cross sections and photoelectron angular distributions agree well with the experimental values of Marr et $a l .{ }^{4}$ and Codling et al., ${ }^{11}$ respectively, as well as with previous Hartree-Fock calculations. ${ }^{12}$ A comparison of these results with those of methane shows that the molecular nature of the $1 t_{2}$ orbital in $\mathrm{CH}_{4}$ leads to photoionization cross sections significantly different from those of the $2 p$ orbital of neon. 


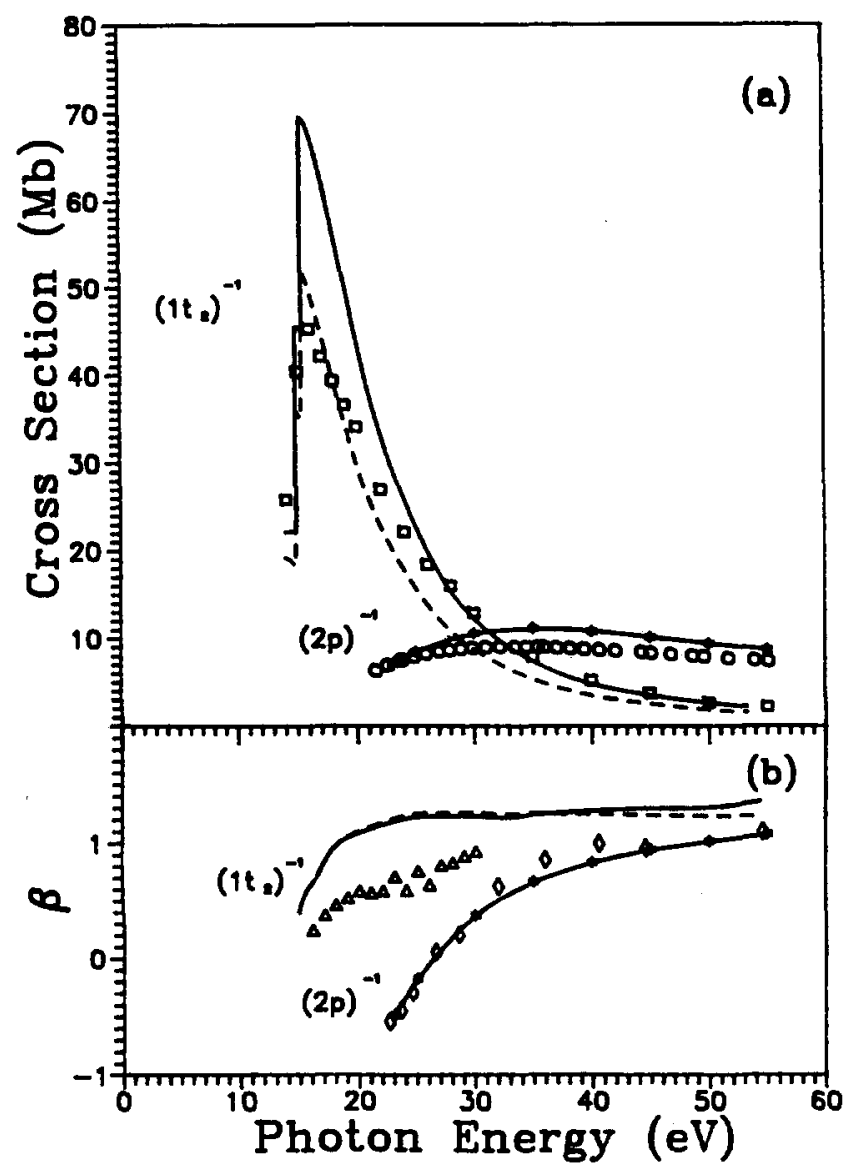

FIG. 1. (a) Cross sections for photoionization of the $1 t_{2}$ orbital of $\mathrm{CH}_{4}$ and the $2 p$ orbital of $\mathrm{Ne}$ : -, present results for $\mathrm{CH}_{4}$ (dipole length); ---, present results for $\mathrm{CH}_{4}$ (dipole velocity). See the Results and Discussion section for discussion of step-like behavior at threshold; $\square$, experimental data of Backx et al. (Ref. 3) for $\mathrm{CH}_{4}$; -*-, present results for $\mathrm{Ne}$ (dipole length); O, experimental data of Marr et al. (Ref. 4) for neon. (b) Photoelectron asymmetry parameters for photoionization of the $1 t_{2}$ orbital of $\mathrm{CH}_{4}$ and the $2 p$ orbital of $\mathrm{Ne}$ : - , present results for $\mathrm{CH}_{4}$ (dipole length); ---, present results for $\mathrm{CH}_{4}$ (dipole velocity); $\Delta$, experimental data of Marr et al. (Ref. 10) for $\mathrm{CH}_{4} ;--$, present results for $\mathrm{Ne} ; \diamond$, experimental data of Codling et al. (Ref. 11) for Ne.

\section{THEORY}

The rotationally unresolved, fixed-nuclei photoionization cross section is given by

$$
\sigma=\frac{4 \pi^{2} \omega}{3 c}\left|\left\langle\Psi_{i}(\mathrm{r} ; R)|\mu| \Psi_{f}(\mathrm{r} ; R)\right\rangle\right|^{2},
$$

where $\mu$ is the dipole moment operator and $\omega$ the photon frequency. In Eq. (1), $\Psi_{i}$ represents the initial state of the molecule with $N$ bound electrons and $\Psi_{f}$ the final state with a photoelectron in the electronic continuum. For the initial state we use a Hartree-Fock wave function. For the final state we assume the frozen-core approximation in which the orbitals of the $(N-1)$ electrons of the molecular ion are taken to be identical to those of the neutral molecule and the photoelectron wave function is a continuum solution of the one-electron Schrödinger equation,

$$
\left[-1 / 2 \nabla^{2}+V_{N-1}(\mathrm{r}, R)-k^{2} / 2\right] \phi_{\mathbf{k}}(\mathbf{r}, R)=0,
$$

where $V_{N-1}(r, R)$ is the static-exchange potential of the mo- lecular ion. The photoelectron orbital $\phi_{\mathbf{k}}$ satisfies appropriate boundary conditions and we have chosen the normalization $\left\langle\phi_{\mathbf{k}} \mid \phi_{\mathbf{k}^{\prime}}\right\rangle=\delta\left(\mathbf{k}-\mathbf{k}^{\prime}\right)$. For ionization of closed-shell targets, $\phi_{k}$ can be assumed orthogonal to the ion orbitals without loss of generality. ${ }^{13}$

To solve Eq. (2) we work with the corresponding integral equation,

$$
\phi_{\mathbf{k}}=\phi_{\mathbf{k}}^{c}+G_{c}^{(-)} U \phi_{\mathbf{k}},
$$

where $\phi_{k}^{c}$ is a pure Coulomb scattering wave, $G_{c}^{(-)}$is the Coulomb Green's function, and $U$ is twice the static-exchange potential with the long-range Coulomb potential of the ionic core removed. The partial wave component $\phi_{k, l h}^{p \mu}$ of $\phi_{\mathrm{k}}$, i.e.,

$$
\phi_{\mathbf{k}}(\mathbf{r})=\left(\frac{2}{\pi}\right)^{1 / 2} \sum_{\substack{p \mu \\ l h}} i^{l} \phi_{k, l h}^{p \mu}(\mathbf{r}) X_{l h}^{p \mu}(\hat{k}),
$$

satisfies its own integral equation,

$$
\phi_{k, l h}^{p \mu}=S_{k, l h}^{p \mu}+G_{c}^{(-)} U \phi_{k, l h}^{p \mu},
$$

where $X_{l h}^{P \mu}(\hat{k})$ is a symmetry-adapted angular basis function, ${ }^{14}$

$$
X_{l h}^{p \mu}(\hat{k})=\sum_{m} b_{l h m}^{p \mu} Y_{l m}(\hat{k}),
$$

and $S_{k . h h}^{p \mu}$ is a corresponding symmetry-adapted partial wave component of the Coulomb function.

We solve Eq. (5) by an iterative procedure which begins by approximating the short-range potential $U$ by a separable potential of the form

$$
\left\langle\mathbf{r}|U| \mathbf{r}^{\prime}\right\rangle=\sum_{\alpha_{i} \alpha_{j}}\left\langle\mathbf{r}|U| \alpha_{i}\right\rangle\left[U^{-1}\right]_{i j}\left\langle\alpha_{j}|U| \mathbf{r}^{\prime}\right\rangle,
$$

where $\alpha_{i}$ and $\alpha_{j}$ are appropriate basis functions, which need not satisfy scattering boundary conditions, and $U_{i j}=\left\langle\alpha_{i}|U| \alpha_{j}\right\rangle$. With this separable approximation for $U$, the solution of Eq. (5) can be written as

$$
\begin{aligned}
\tilde{\phi}_{k, l h}^{p \mu}(\mathbf{r})= & S_{k, l h}^{p \mu}(\mathbf{r})+\sum_{\alpha_{r} \alpha_{j}}\left\langle\mathbf{r}\left|G_{c}^{(-)} U\right| \alpha_{i}\right\rangle\left[D^{-1}\right]_{i j} \\
& \times\left\langle\alpha_{j}|U| S_{k, l h}^{p \mu}\right\rangle,
\end{aligned}
$$

where

$$
D_{i j}=\left\langle\alpha_{i}\left|U-U G_{c}^{(-)} U\right| \alpha_{j}\right\rangle .
$$

Equation (8) provides an approximate solution $\tilde{\phi}_{k, l h}^{p \mu}$ to the $\phi_{k, l h}^{p \mu}$ of Eq. (5). We have developed an iterative procedure for obtaining converged solutions of Eq. (5). ${ }^{13}$ To ensure convergence of the photoionization cross sections, we used photoelectron orbitals obtained after three steps in this iterative procedure.

\section{COMPUTATIONAL DETAILS}

\section{Methane}

The ground state SCF wave function of methane, with an electronic configuration $1 a_{1}^{2} 2 a_{1}^{2} 1 t_{2}^{6}\left({ }^{1} A_{1}\right)$, was obtained in a $(9 s 5 p, 4 s) /[4 s 3 p, 3 s]$ Cartesian Gaussian basis of Dunning. ${ }^{15}$ For tetrahedral geometry and an equilibrium $\mathbf{C}-\mathbf{H}$ bond distance of 2.0503 a.u., our basis gives an SCF energy 
of -40.185831 a.u. For the basis functions $\left\{\alpha_{i}\right\}$, in Eq. (8) we chose Cartesian Gaussian functions defined by

$$
\begin{aligned}
\phi^{\alpha, l, m, n, A}(\mathbf{r})= & N\left(x-A_{x}\right)^{l}\left(y-A_{y}\right)^{m}\left(z-A_{z}\right)^{n} \\
& \times \exp \left(-\alpha|\mathbf{r}-\mathbf{A}|^{2}\right) .
\end{aligned}
$$

The basis sets used in solving for the photoelectron continuum orbitals are listed in Table I. All matrix elements arising in the solution of Eq. (8) were evaluated using single-center expansions about the carbon atom. The partial wave expansion of the photoelectron orbital, Eq. (4), was truncated at $l_{p}$ $=7$. Other parameters used in the required partial wave expansions were as follows:

(i) $l_{m}=$ maximum $l$ included in the expansion of the scattering functions and of the Coulomb Green's function $=7$;

(ii) $l_{s}^{\text {ex }}=$ maximum $l$ included in the expansion of the scattering function in the exchange terms $=7$;

(iii) $l_{i}^{\text {ex }}=$ maximum $l$ included in the expansion of the occupied orbitals in the exchange terms $=7$;

(iv) $l_{i}^{\text {dir }}=$ maximum $l$ included in the expansion of the occupied orbitals in the static potential $=7$;

(v) $\lambda_{m}^{\mathrm{ex}}=$ maximum $l$ included in the expansion of $1 /$ $r_{12}$ in the exchange terms $=7$;

(vi) $\lambda \underset{m}{\text { dir }}=$ maximum $l$ included in the expansion of $1 /$ $r_{12}$ in the static potential $=13$.

The grid for quadrature of the radial integrals consisted of 400 points and extended to 44.8 a.u. with a step size varying from 0.01 a.u. near the origin up to 0.64 a.u. at larger distances.

It is convenient to carry out these calculations in the $C_{2 V}$ symmetry group. In these calculations we assume the orientation of the $\mathbf{C H}_{4}$ molecule to be as illustrated in Fig. 2. Accordingly, the triply degenerate $1 t_{2}$ orbital transforms to $b_{2}, b_{1}$, and $a_{1}$ orbitals. The photoionization cross section is then obtained by summing over the dipole-allowed

TABLE I. Basis sets used in obtaining photoelectron orbitals from Eq. (8).

\begin{tabular}{cccc}
\hline \hline $\begin{array}{c}\text { Continuum } \\
\text { symmetry }\end{array}$ & Center & $\begin{array}{c}\text { Type of Gaussian } \\
\text { function }\end{array}$ & Exponents \\
\hline Methane & & & \\
$\mathrm{A} 1$ & $\mathrm{C}$ & $s$ & $5.4,1.8,0.6,0.2$ \\
& $\mathrm{C}$ & $z$ & $1.2,0.4$ \\
& $\mathrm{H} 1 ; \mathrm{H} 2$ & $s$ & $1.2,0.4$ \\
& $\mathrm{H} 3 ; \mathrm{H} 4$ & $s$ & $1.2,0.4$ \\
$\mathrm{~A} 2$ & $\mathrm{C}$ & $x y$ & $7.2,2.4,0.8,0.27$ \\
& $\mathrm{H} 1 ; \mathrm{H} 2$ & $x$ & $1.2,0.4$ \\
& $\mathrm{H} 3 ; \mathrm{H} 4$ & $y$ & $1.2,0.4$ \\
$\mathrm{~B} 1$ & $\mathrm{C}$ & $y$ & $7.2,2.4,0.8,0.27$ \\
& $\mathrm{H} 1 ; \mathrm{H} 2$ & $s$ & $1.2,0.4$ \\
& $\mathrm{H} 3 ; \mathrm{H} 4$ & $y$ & $1.2,0.4$ \\
B2 & $\mathrm{C}$ & $x$ & $7.2,2.4,0.8,0.27$ \\
& $\mathrm{H} 1 ; \mathrm{H} 2$ & $x$ & $1.2,0.4$ \\
& $\mathrm{H} 3 ; \mathrm{H} 4$ & $s$ & $1.2,0.4$ \\
Neon & & & $16.0,8.0,4.0,2.0$ \\
$s$ & $\mathrm{Ne}$ & $s$ & $1.0,0.5$ \\
& & & $16.0,8.0,4.0,2.0$ \\
$d$ & $\mathrm{Ne}$ & $d$ & $1.0,0.5$ \\
\hline \hline
\end{tabular}

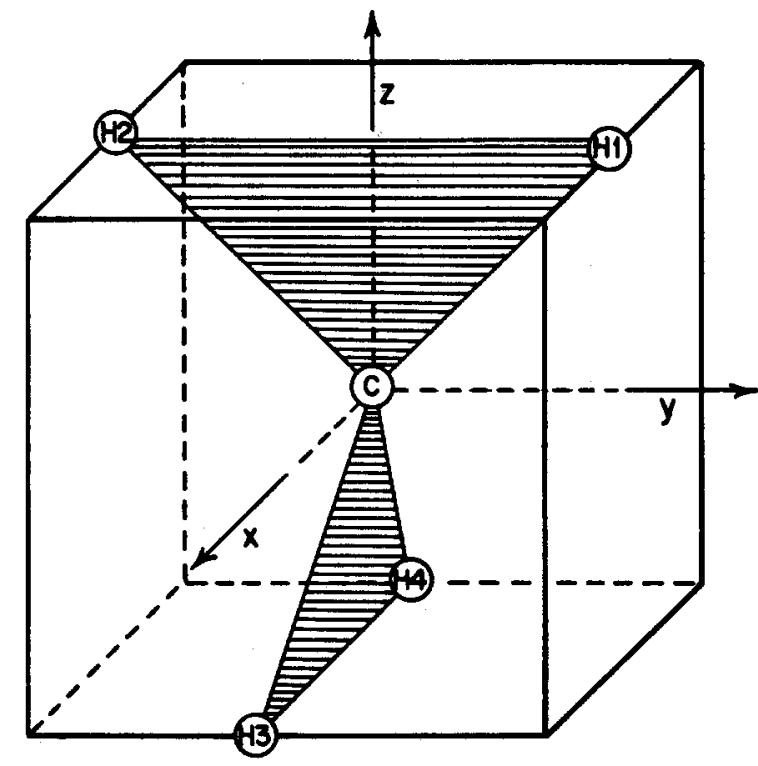

FIG. 2. Orientation of methane assumed in calculations.

components: $\quad b_{2} \rightarrow k a_{1}, k a_{2}, k b_{2} ; \quad b_{1} \rightarrow k a_{1}, k a_{2}, k b_{1} ; \quad$ and $a_{1} \rightarrow k a_{1}, k b_{1}, k b_{2}$.

As a confidence check on the computer codes used in these calculations and to assess the convergence of the corresponding partial wave expansions, we obtained cross sections for photoionization of the $1 b_{2}$ bonding orbital of linear $\mathrm{H}_{2} \mathrm{O}$ with procedures we have used previously for many linear molecules. ${ }^{6}$ The partial wave expansions used in these "linear" codes were approximately four times as large as those in our present calculations on methane. The resulting cross sections and asymmetry parameters and those obtained for $\mathrm{H}_{2} \mathrm{O}$ with a bond angle of $179.9^{\circ}$ using our present procedures, with their smaller partial wave expansions, were nearly identical.

\section{RESULTS AND DISCUSSION}

Figure 1 (a) shows our calculated photoionization cross sections for the $1 t_{2}$ orbital of methane along with the experimental data of Backx et al. ${ }^{3}$ The cross sections shown here, in both the length and velocity form, were calculated at the equilibrium internuclear geometry of methane and assume that the photoelectron spectrum is made up of three electronic bands with maxima at $13.5,14.5$, and $14.8 \mathrm{eV}$, respectively resulting from the Jahn-Teller distortion of the ${ }^{2} T_{2}$ ion of $\mathrm{CH}_{4}{ }^{9}{ }^{9}$ The actual cross sections used in each of these intervals were obtained from the fixed-nuclei ${ }^{2} T_{2}$ ion calculation. This procedure builds in step-like behavior very near threshold and gives essentially the same cross sections above $\sim 18$ $\mathrm{eV}$ as obtained assuming a single ionization threshold of 12.6 $\mathrm{eV}$. From 14 to $16 \mathrm{eV}$ photon energy there is a sharp increase in the $1 t_{2}$ experimental cross section from 25.8 to $45.2 \mathrm{Mb}$. Our calculations show the same sharp rise as experiment, suggesting that this behavior arises from successive vibronic contributions associated with Jahn-Teller components of the ion. The raw fixed-nuclei cross sections without any such assumed threshold behavior, which we do not show in Fig. 1 (a), agree well with the results obtained by Cacelli $e t a l^{8}$ 
using a one-center representation of the $\mathrm{CH}_{4}$ wave function and the Stieltjes imaging technique. With a resolution of $\sim 1$ $\mathrm{eV}$, the experimental data shown in Fig. 1(a) does not resolve any vibrational structure associated with the JahnTeller distorted components of the methane ion. Experiments with sufficient resolution to resolve such structure near threshold would provide useful insight into the behavior of these cross sections at low energy. Above a photon energy of $16 \mathrm{eV}$ our calculated cross sections agree well with the experimental data.

Figure 1(a) also shows experimental cross sections for $2 p$ photoionization of neon. While the methane $1 t_{2}$ cross section rises steeply at threshold and decreases dramatically over an energy range of $40 \mathrm{eV}$, the neon $2 p$ cross section remains relatively flat through the same energy range, with a weak maximum about five times smaller in magnitude than in the $1 t_{2}$ cross section. The molecular nature of the $1 t_{2}$ orbital clearly gives rise to significantly different photoionization cross sections from those of the $2 p$ orbital of neon.

Our calculated $1 t_{2}$ photoelectron asymmetry parameters are compared with the measured values of Marr et al. ${ }^{10}$ in Fig. 1(b). These results were obtained by averaging the values of the asymmetry parameters obtained by again assuming that the spectrum is a composite of three electronic bands associated with the Jahn-Teller components of the distorted ion of $\mathrm{CH}_{4}$. The calculated photoelectron asymmetry parameters are larger than the measured values which are only available up to $30 \mathrm{eV}$. The present results are, however, in substantially better agreement with experiment than those of earlier orthogonalized plane-wave calculations. ${ }^{16}$ Measurements above $30 \mathrm{eV}$ would be useful in assessing the quality of our calculated values in the higher energy range.

\section{Neon}

For neon we used the Hartree-Fock wave function constructed from the (10s7p) Cartesian Gaussian basis set of Huzinaga et al. ${ }^{17}$ which gives an energy of -128.538079 a.u. The numerical grid consisted of 400 points and extended to 28 a.u. with a step size of 0.01 a.u. out to 1.0 a.u., the largest step size being 0.16 a.u. The Cartesian Gaussian basis $\left\{\alpha_{i}\right\}$ used in these calculations is shown in Table $I$.

Figure 1(a) shows our calculated $2 p$ photoionization cross section along with the experimental data of Marr et al. ${ }^{4}$ The cross sections and photoelectron angular distributions obtained with the length and velocity forms for neon are essentially equivalent and, for convenience, only the length forms are shown in Figs. 1(a) and 1(b). Our calculated cross sections for neon, as well as those of similar calculations, ${ }^{12}$ compare well with experiment. Calculations beyond the Hartree-Fock level provide cross sections in better agreement with the experimental values. ${ }^{18,19}$ Our results show the same significant difference in shape and magnitude between the $2 p$ and $1 t_{2}$ cross sections of neon and methane, respectively, as is seen experimentally.

The calculated asymmetry parameters for $2 p$ photoionization of neon and the experimental data of Codling et al. ${ }^{11}$ are shown in Fig. 1 (b). The agreement between our calculated results and the experimental values is good.

\section{CONCLUDING REMARKS}

We have studied the cross sections and asymmetry parameters for photoionization of the $1 t_{2}$ orbital of $\mathrm{CH}_{4}$ using static-exchange continuum orbitals of $\mathrm{CH}_{4}^{+}$to represent the photoelectron wave function. Threshold structure was incorporated into these calculated cross sections by assuming that the photoelectron spectrum is made up of three electronic bands arising from Jahn-Teller distortions and having maxima at $13.5,14.5$, and $14.8 \mathrm{eV}$. The resulting cross sections, including the sharp rise near threshold, agree well with the experimental data. Measurements near threshold with sufficient photoelectron energy resolution to resolve vibrational structure associated with these Jahn-Teller distortions of the methane ion would provide useful insight into the behavior of these cross sections. The agreement between our calculated asymmetry parameters and the experimentally measured values is less satisfactory.

\section{ACKNOWLEDGMENTS}

This material is based upon research supported by the National Science Foundation under Grant No. CHE8521391 and the U. S.-Brazil Science Cooperative Program under Grant No. INT82-19691. We also acknowledge use of the resources of the San Diego SuperComputer Center. The authors thank Dr. J. A. Stephens for many helpful discussions, and for making available results of earlier multiple scattering calculations on methane.

'See, for example, R. A. Buckingham, H. S. W. Massey, and S. R. Tibbs, Proc. R. Soc. A 178, 119 (1941).

${ }^{2}$ A. Dalgarno, Proc. R. Soc. London Ser. A 65, 663 (1952).

${ }^{3}$ C. Backx and M. J. van der Wiel, J. Phys. B 8, 3020 (1975).

${ }^{4}$ G. V. Marr and J. B. West, At. Data Nucl. Data Tables 18, 497 (1976).

'See, for example, D. Lynch, V. McKoy, and R. R. Lucchese, in Symposium on Resonances in Electron-Molecule Scattering, van der Waals Complexes, and Reactive Chemical Dynamics, edited by D. G. Truhlar, ACS Symp. Ser. 263 (American Chemical Society, Washington, D.C., 1984).

${ }^{6}$ R. R. Lucchese, K. Takatsuka, and V. McKoy, Phys. Rep. 131, 147 (1986).

'M. Braunstein, V. McKoy, L. E. Machado, and M. A. P. Lima, J. Chem. Phys. ( to be published).

${ }^{8}$ I. Cacelli, V. Carravetta, and R. Moccia, J. Phys. B 18, 1375 (1985).

'See, for example, J. W. Rabalais, T. Bergmark, L. O. Werme, L. Karlsson, and K. Siegbahn, Phys. Scr. 3, 13 (1971); A. W. Potts and W. C. Price, Proc. R. Soc. London Ser. A 326, 165 (1972); R. N. Dixon, Mol. Phys. 20, 113 (1971); and more recently M. N. Paddon-Row, D. J. Fox, J. A. Pople, K. N. Houk, and D. W. Pratt, J. Am. Chem. Soc. 107, 7696 (1985).

${ }^{10}$ G. V. Marr and R. M. Holmes, J. Phys. B 13, 939 (1980).

${ }^{11}$ K. Codling, R. G. Houlgate, J. B. West, and P. R. Woodruff, J. Phys. B 9, L83 (1976).

${ }^{12}$ D. J. Kennedy and S. T. Manson, Phys. Rev. A 5, 227 (1972).

${ }^{13}$ R. R. Lucchese, G. Raseev, and V. McKoy, Phys. Rev. A 25, 2572 (1982).

${ }^{14}$ P. G. Burke, N. Chandra, and F. A. Gianturco, J. Phys. B 5, 2212 (1972).

${ }^{15}$ T. H. Dunning, J. Chem. Phys. 53, 2823 (1970).

${ }^{16} \mathrm{~J}$. W. Rabalais, Principles of Ultraviolet Photoelectron Spectroscopy (Wiley-Interscience, New York, 1977), p. 187.

${ }^{17}$ S. Huzinaga, Gaussian Basis Sets for Molecular Calculations (Elsevier, New York, 1984), p. 83.

${ }^{18}$ M. Ya Amusia, N. A. Cherepkov, and L. V. Chernyshava, JETP 33, 90 (1971).

${ }^{19}$ P. G. Burke and K. T. Taylor, J. Phys. B 8, 2620 (1975). 